\title{
NIH clarifies grant money policy
}

On 26 January 2007, the National Institutes of Health (NIH) issued a notice ${ }^{1}$ explaining that NIH grant monies cannot be used for work with live vertebrate animals unless that work is associated with a valid Animal Welfare assurance from the Office of Laboratory Animal Welfare (OLAW) and/or a valid IACUC-approved protocol. The Office of Management and Budget Cost Principles and the NIH Grants Policy Statement (NIHGPS) do not allow for the animal work to be charged against NIH grants in cases when either or both of these conditions are not met.

The NIH notice listed the following as specific situations under which charges are not allowed:

- "The conduct of animal activities in the absence of a valid Assurance on file with OLAW.
- "The conduct of animal activities in the absence of a valid IACUC approval of the activity. Absence of IACUC approval includes failure to obtain IACUC approval, expiration, or suspension of IACUC approval ${ }^{1}$."

Any situations that do not meet these terms and conditions as defined in the NIHGPS should be reported to the Institute or Center supporting the grant. During periods when there is no valid Assurance or IACUC approval, grantees must continue to care for their animals, although whether grant money will be available to go toward such animal care activities will only be decided on a case-by-case basis.

The notice specifies that the abovementioned situations constitute serious noncompliance with the Public Health Service Policy on Humane Care and Use of
Laboratory Animals ${ }^{2}$ that must be promptly reported to OLAW.

In addition, the notice includes a reminder that the primary grantee is responsible for the conduct of a project involving institutional collaboration, including grant expenditures by all parties. As such, the primary grantee must ensure that collaborating organizations have valid Assurances and IACUC approvals for the animal work they are conducting.

1. NIH. NOT-0D-07-044. NIH Policy on Allowable Costs for Grant Activities Involving Animals when Terms and Conditions are not Upheld. (26 January 2007). http://grants.nih.gov/ grants/guide/notice-files/NOT-0D-07-044. html.

2. Public Health Service. Policy on Humane Care and Use of Laboratory Animals IV.F.3 (US Department of Health and Human Services, Washington, DC, 1986; reprinted 2002).

\section{New BMBL edition issued}

In February 2007, the US Department of Health and Human Services, Centers for Disease Control and Prevention, and National Institutes of Health published the 5 th edition of Biosafety in Microbiological and Biomedical Laboratories (BMBL). The BMBL provides guidelines applicable to both in vitro and in vivo work with human and zoonotic pathogens.

The BMBL includes 'agent summary statements' that describe the appropriate biosafety levels for working with various microorganisms, as well as recommendations for proper handling, safety equipment, and facility safeguards that should be in place when conducting research on particular agents. Agent summary statements are included for microorganisms that fit the following criteria:

- "the agent is a proven hazard to laboratory personnel working with infectious materials;

\section{REGULATION UPDATE}

\section{Bill aims to address veterinarian shortage}

The 'Veterinary Public Health Workforce Expansion Act of 2007' (S 746/HR 1232), jointly introduced by Senator Wayne Allard (R-CO) and Congresswoman Tammy Baldwin (D-WI) on 2 March 2007, would establish a federal grant program to increase the number of veterinarians trained in several critical areas of public health.

Evidence suggests there are serious shortages in veterinarians trained in such specialties as biomedical research, food safety, and bioterrorism preparedness. The new legislation would increase the research, diagnostic, and training capacity of the nation's 28 veterinary medical colleges.
- "the agent has a high potential for causing [laboratory-associated infections] even though no documented cases exist;

- "the agent causes grave disease or presents a significant public health hazard ${ }^{1}$."

Updates since the last edition include the addition of new or updated agent summary statements for those agents now classified as 'Select Agents', including an updated Agent Summary Statement for influenza viruses, and a new chapter on biosecurity, defined as "the discipline addressing the security of microbiological agents and toxins and the threats posed to human and animal health, the environment, and the economy by deliberate misuse or release ${ }^{1}$."

1. DHHS, CDC \& NIH. Biosafety in Microbiological and Biomedical Laboratories, 5th edn. (US Government Printing Office, Washington, DC, 2007). http://www.cdc.gov/OD/OHS/biosafety/ bmbl5/bmbl5toc.htm. 